microscopy showed that the dried products prepared by both acid and base catalysis consist of aggregates of fibers $\sim 200 \mu \mathrm{m}$ long and 150-600 $\mathrm{nm}$ in diameter. Similar fiber aggregates were observed for the assemblies produced by the organogelator alone, proving its role as the template. The fibers produced by acid-catalyzed polymerization were smooth, while those produced by basecatalyzed polymerization had rough surfaces. This difference was ascribed to the production of $\mathrm{TiO}_{2}$ particles during basecatalyzed polymerization.

Unlike the dried product, the calcined samples exhibited fiber structures only when the polymerization was basecatalyzed. This result is consistent with the presence of anionic propagation species during base-catalyzed sol-gel polymerization. These negatively charged intermediates adsorb onto the positively charged organogelator template, and the polymerization proceeds along the template. Transmission electron micrographs of the calcined base-catalyzed product show that the nanofibers are hollow and have internal diameters of 50-150 nm. X-ray diffraction studies of the calcined nanofibers show that they have the desired anatase structure.

GREGORY KHITROV

\section{Low-Temperature Process Produces Microcanals for Microfluidic Chips}

Researchers at Sandia National Laboratories have developed a microchip processing technique that creates raised microscopic canals on chips, through which liquids or gases can flow from one chip feature to another. The raised hemispherical canals, 8-100 $\mu \mathrm{m}$ in diameter, have been created on silicon, glass, and quartz surfaces. Some of the canals have been made with curvatures with radii as small as $8 \mu \mathrm{m}$. These canals can be small enough and curvy enough that some liquids or gases pass easily through them and others pass more slowly. This ability to distinguish among fluidic materials is useful for chemical-separation applications, the most common use of microfluidic devices.

To make the canals, the researchers pattern a thin layer of photoresist on the wafer's surface using a conventional photo mask and light, then develop away areas of photoresist exposed to the light, leaving a network of photoresist ridges on the wafer's surface that eventually becomes the canals' interiors.

Next, they heat the wafer to a relatively low temperature of $100^{\circ} \mathrm{C}$ for about $20 \mathrm{~s}$, which causes the square-edged ridges to slump into a hemispherical shape. A 2- $\mu \mathrm{m}-$

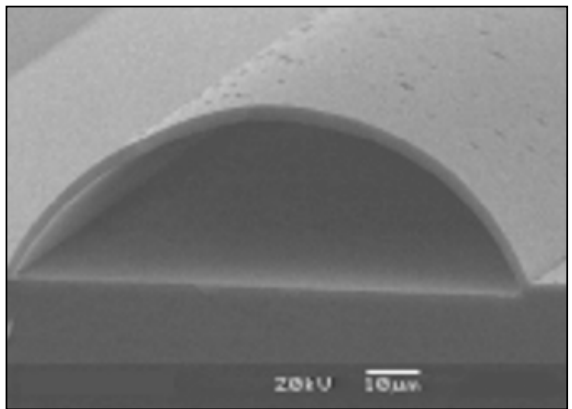

Radius of curvature: $52 \mu \mathrm{m}$

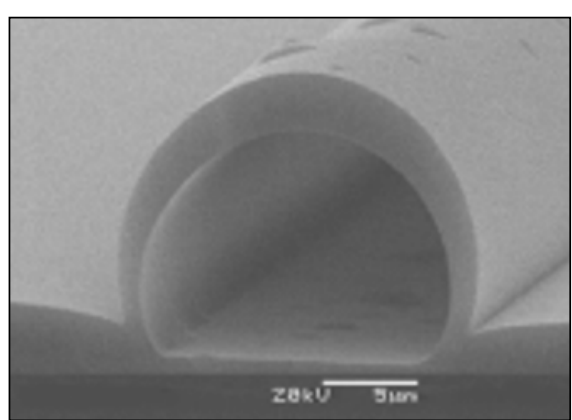

Radius of curvature: $8 \mu \mathrm{m}$

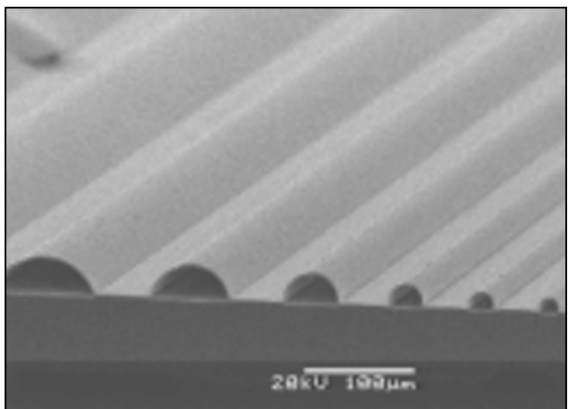

Microscopic views of raised hemispherical canals 8-100 $\mu \mathrm{m}$ in diameter.

thick film of silicon oxynitride is deposited over the rounded photoresist, and the entire wafer is soaked in an acetone bath until the remaining photoresist is dissolved, leaving hollow tunnels on the wafer's surface.

The traditional trench-and-seal method involves the joining of two pre-trenched wafers. But the intense heat required for bonding - up to $1000^{\circ} \mathrm{C}$ - can damage other chip features, and the care required to remove contaminant particles from the two halves increases the difficulty and cost of manufacturing.

The newly developed and patented technique is 10 to 100 times faster than the trench-and-seal method, said co-developer Carol Ashby. She said that the resulting tunnels are virtually indestructible.

Co-developer Carolyn Matzke of Sandia's Compound Semiconductor
Research Laboratory said that the technique's compatibility with standard semiconductor batch-processing tools should allow future microfluidic devices to be made quickly and cheaply in a microchip factory.

\section{Calculations Indicate that 2D Photonic Crystals Based upon Archimedean-Like Tilings Have Nearly Isotropic Properties}

Two-dimensional (2D) artificial crystals with photonic-band structures can be used for applications such as microwave or millimeterwave filters for $\mathrm{rf}$ photonics or for suppression of spontaneous emission in microcavity resonators. However, 2D photonic crystals based upon real 2D crystals belong to one of the five 2D Bravais lattices. These crystals possess few symmetry elements and are too few to achieve ideal optical isotropy. In calculations performed by S. David, A. Chelnokov, and J.-M. Lourtioz of the Institut d'Electronique Fondamentale at the Université Paris-Sud, three Archimedean-like 2D tiling configurations were considered. Each of these configurations displayed 12-fold symmetry, making the resulting 2D crystal nearly photoisotropic, as they reported in the July 15 issue of Optics Letters.

Complete tiling of a plane with a single type of regular polygon can only occur for squares, triangles, and hexagons. However, combinations of squares, triangles, and hexagons can be used to completely tile a plane. The researchers described the Archimedean tilings as "regular convex polygons that are not necessarily identical, but are identically arranged around each vertex." The five 2D Bravais lattices are a subcategory of Archimedean tilings. Three tilings used in this experiment are composed of squares and equilateral triangles. Atoms are then assigned to either square or triangular Bravais lattices, thus allowing definition of Wigner cells. This enabled the team to investigate these theoretical entities with numerical methods, generating information on their band structure and gaps as a function of lattice direction.

Theoretical diffraction patterns of these systems are generated with Fourier transform calculations. The result is a set of points that outlines the vertices of a dodecagon, implying 12-fold symmetry. The plane-wave method was used to construct band diagrams for these crystals. Calculations were performed on main crystallographic directions for both transverse electric (TE) and transverse magnetic (TM) polarizations. These results were 
then compared with transmission spectra results generated using a finite-element method. The agreement between the plane-wave method and finite-element calculations was very good.

Results from this experiment showed that 12 -fold symmetry structures had bandgaps that are nearly independent of propagation direction. As these tilings increase in complexity, so do their number of symmetry elements, rendering them more isotropic.

JUNE LAU

\section{Pulse-Generation Limited to Five Optical Cycles Using Negatively Chirped Optical Parametric Oscillator}

Researchers at the University of St. Andrews in the United Kingdom have demonstrated the feasibility of using optical parametric oscillators (OPOs) with chirped grating crystals to produce mid-infrared pulses with a bandwidth as narrow as five optical cycles. In the July 15 issue of Optics Letters, T. Beddard, M. Ebrahimzadeh, T.D. Reid, and W. Sibbett report that crystals of aperiodically poled lithium niobate (APPLN) effectively recompressed dispersionbroadened laser pulses of known frequency, resulting in the bandwidth restriction. The recompression is due to the chirped grating period of the crystals.

A 1-mm-long APPLN crystal with eight different linearly chirped gratings was used in the study. The least-chirped grating had a uniform period of $22.96 \mu \mathrm{m}$, while the most-chirped had a period ranging from $21.89 \mu \mathrm{m}$ to $23.55 \mu \mathrm{m}$. The average grating period was constant throughout the series, and the crystal was studied in both positively and negatively chirped orientations. When the crystal was pumped collinearly by a Ti:sapphire laser, the OPO generated nearly transform-limited 53-fsduration pulses at a center wavelength of $3 \mu \mathrm{m}$ in the best-case scenario. This optimum output (approximately five optical cycles at FWHM intensity) was found to correspond to a negatively chirped grating with periods from $22.00 \mu \mathrm{m}$ to $23.54 \mu \mathrm{m}$. Pulses emanating from the positively chirped OPO orientation were consistently broader. The development of numerical models to explain the observations is planned.

TIM PALUCKA

\section{Sol-Gel Coating Improves Structural Stability of $\mathrm{LiCoO}_{2}$ Cathodes during Electrochemical Cycling}

A team of researchers from Samsung SDI Company and Seoul National Univer- sity has greatly improved the structural stability of $\mathrm{LiCoO}_{2}$ cathodes by sol-gel coating $\mathrm{LiCoO}_{2}$ powders with $\mathrm{SnO}_{2}$. The coated materials were heat-treated to $400^{\circ} \mathrm{C}$ or $500^{\circ} \mathrm{C}$ and retained $87 \%$ and $84 \%$ of their initial capacities after 47 cycles between voltages of $4.4 \mathrm{~V}$ and $2.75 \mathrm{~V}$. This was attributed to a nonuniform Sn distribution throughout the particles and the resulting suppression of the monoclinicto-hexagonal phase transition commonly observed in uncoated materials upon cycling.

Lithium transition-metal oxides are used as cathode materials for lithium-ion cells. The most stable among the $\alpha-\mathrm{NaFeO}_{2}$ structure types is $\mathrm{Li}_{1-x} \mathrm{CoO}_{2}$.

According to the scientists, " $\mathrm{Li}_{1-x} \mathrm{CoO}_{2}$ cycling stability is excellent over the limited composition range $0<x<0.5$, but the stability rapidly deteriorates for $x>0.5 . "$ They attribute this behavior to the occurrence of a monoclinic-to-hexagonal phase transition upon cycling at $x \sim 0.5$, which is accompanied by a large decrease in the $c$ axis dimension, while the $a$-axis length remains almost unchanged. The resulting differential stress leads to fracture of the oxide material. Efforts to improve the cycling stability of lithium cobalt oxide electrodes by partial substitution of $\mathrm{Al}$ or $\mathrm{Mg}$ on the Co site were unsuccessful, despite the fact that the change in $c$-axis dimensions was much smaller than in undoped materials. This influenced the researchers to use an alternate approach. They said that surface doping with metal oxides can sometimes suppress phase transitions.

Their procedure started with a $10-\mu \mathrm{m}$ $\mathrm{Li}_{1-x} \mathrm{CoO}_{2}$ powder prepared by a solidstate method. As reported in the August issue of Electrochemical and Solid-State Letters, the grains were then subjected to sol-gel coating in a solution of tin(IV)ethylhexanoisopropoxide in isopropanol and subsequently dried at $150^{\circ} \mathrm{C}$. Parts of this coated sample were heated to 400, 500, and $600^{\circ} \mathrm{C}$, respectively. X-ray diffraction (XRD) patterns confirmed that a single phase with the $\alpha-\mathrm{NaFeO}_{2}$ structure was formed in all samples. Lattice-constant analysis proved that the $\mathrm{SnO}_{2}$ was present as an amorphous surface layer in the $150^{\circ} \mathrm{C}$ material, whereas changes in the lattice constants of the samples treated at higher temperatures implied the formation of a $\mathrm{LiCo}_{1-x} \mathrm{Sn}_{x} \mathrm{O}_{2}$ solid-solution phase. Electron microprobe analysis showed increasing migration of the $\mathrm{Sn}$ atoms into the grains with increasing heating temperature, and the $600^{\circ} \mathrm{C}$ material exhibited a homogenous distribution throughout the grains. Upon cycling, the materials with a nonuniform $\mathrm{Sn}$ distribution (e.g., the $400^{\circ} \mathrm{C}$ and $500^{\circ} \mathrm{C}$ samples) had lower initial capacities than uncoated $\mathrm{LiCoO}_{2}$, but retained $87 \%$ and $84 \%$ of it after 47 cycles as stated earlier, whereas the uncoated and $600^{\circ} \mathrm{C}$ samples showed only $49 \%$ of their initial capacity after the same number of cycles. Cyclic voltammograms revealed that the $400^{\circ} \mathrm{C}$ and $500^{\circ} \mathrm{C}$ samples did not undergo the monoclinicto-hexagonal phase transition that was observed between $4.15 \mathrm{~V}$ and $4.2 \mathrm{~V}$ for the $150^{\circ} \mathrm{C}$ and $600^{\circ} \mathrm{C}$ materials. Ex situ XRD experiments showed that this phase transition induced cation disorder in the oxides by the exchange of $\mathrm{Li}$ and $\mathrm{Co}$ between their respective sites.

The researchers concluded, "The higher Sn concentrations at the particle surfaces prevent the phase transition from the monoclinic to the hexagonal phase."

CORA LIND

\section{Host-Directed Polar Order Observed in Organic Molecular Crystals}

Polar order in organic inclusion compounds is normally the result of direct head-to-tail contact between the guest molecules and is not affected by the host lattice. Another type of organic inclusion compound that exhibits polar order as a result of guest-host interactions has been discovered by graduate student Jennifer Swift and Prof. Michael Ward at the Department of Chemical Engineering and Materials Science at the University of Minnesota. The host structure consists of hydrogen-bonded guanidinium-ion "sheets" connected by perpendicular 4,4'-biphenyldisulfonate (BPDS) ion "pillars." The guest molecules are trapped in pores whose boundaries are defined by the guanidinium sheets on the top and bottom and the BPDS pillars on the sides. Molecular crystals exhibiting polar order were prepared using 4-nitrooxylene, 1-nitro-, 1-iodo-, and 1-cyanonapthalene (NX, NN, IN, and $\mathrm{CN}$, respectively) as guest species.

As reported in the August issue of Chemistry of Materials, the inclusion compounds were synthesized in nearly quantitative yield by preparing a methanol solution of equimolar guanidine hydrochloride and 4,4'-biphenyldisulfonic acid along with excess guest, and allowing the solvent to slowly evaporate at room temperature. Real-time, in situ atomic force microscopy studies showed that the crystal forms by means of layer-by-layer growth.

The scientists assert that the high degree of polar order exhibited by the guest molecules is the result of ion-dipole interactions between the host guanidinium ions in the bottom sheet of each layer and the guest $\mathrm{C}-\mathrm{X}\left(\mathrm{X}=\mathrm{NO}_{2}, \mathrm{I}, \mathrm{CN}\right)$ group dipoles during 